\title{
Erfahrungen mit dem Schulfach Geographie
}

Im Vergleich mit anderen Schulfächern zeichnet sich die Geographie vor allem durch die große Mannigfaltigkeit des zu behandelnden Stoffes aus. Sie beschäftigt sich mit materiellen wie mit geistigen Erscheinungen unterschiedlichster Art, und sie hat erst noch Rücksicht zu nehmen auf alle die vielfältigen Varianten im Bereich der ganzen Erdoberfläche. Diese Besonderheit des Schulfaches Geographie kann je nach Standpunkt als Belastung oder als Vorzug empfunden werden. Eine Belastung bedeutet sie sicher insofern, als überdurchschnittliche Anstrengungen zu ihrer methodischen Bewältigung erforderlich sind. In den Augen vieler mag die Geographie zwar als eher einfaches Schulfach gelten, für den Lehrer ist sie es nicht. Die stoffliche Mannigfaltigkeit hat aber den Vorzug, den Blick für das Ganze der uns umgebenden Welt zu schulen. Weil die unterschiedlichsten Stoffbereiche geographisch relevant sein können, müssen zu ihrer Bearbeitung vergleichbare Nenner auf übergeordneter Ebene gesucht werden, und in dieser Betätigung liegt der besondere Wert des Faches. Gerade die ganzheitliche und häufig erdumspannende Problematik der heutigen Zeit sollte eigentlich die Dringlichkeit dieser Art von Schulung zeigen. Die für den Unterricht negative Seite der stofflichen Mannigfaltigkeit der Geographie verliert im Blick auf den positiven Aspekt an Gewicht. Im positiven Aspekt, in der Eignung des Faches für Übungen in der Zusammenschau, im Erkennen von Relationen und zum Teil schicksalshaften Gemeinsamkeiten von an sich unterschiedlichen Sachbereichen, kann sogar das eigentliche Wesen des Schulfaches Geographie gesehen werden.

\section{Ein Gesamtziel}

Es ist nicht so, wie vielleicht vermutet werden könnte, daß aus der Not eine Tugend gemacht wird, wenn das Wesen der Geographie gerade aus ihrer uneinheitlichen Stoffülle hergeleitet wird. Tatsächlich ist nämlich diese Stoffülle, der Aufgabe der Geographie entsprechend, nicht nur einfach vorhanden, sondern sie ist die notwendige Substanz zur Lösung der geographischen Aufgabe, und die Lösung fällt um so besser aus, je mehr stoffliche
Details mitberücksichtigt werden können. Entscheidend ist nun allerdings erst die Feststellung, da $\beta$ alle stoffliche Substanz immer nur unter einem bestimmten Gesichtspunkt, eben dem geographischen, relevant wird. Die einzelnen Stoffgebiete selber kommen ja zum Teil bereits in anderen Fächern zur Darstellung, und wenn in der Schule noch mehr Fächer erteilt würden, wäre sogar aller Stoff schon einmal an andere Fächer vergeben. Die Geographie beginnt aber gerade dort wirklich Geographie zu sein, wo ein bestimmter Stoff nicht mehr, wie in den anderen Fächern, für sich, sondern als Baustein des Ganzen der geographischen Welt angeschaut wird, wo der Stoff nicht mehr selber wichtig ist, wo vielmehr sein Beitrag an das Ganze Bedeutung erhält. Das Fach Geographie hat den Auftrag, den dingerfüllten irdischen Raum im Zusammenhang und nicht mit der Betonung auf der Eigenart der stofflichen Elemente zu zeigen. Dargestellt werden muß der geographische Raum als Raum. Dieser Raum ist wohl kein geometrischer, neutraler Raum, sondern ein stofflicher Raum, aber der Stoff ist nur soweit von Interesse, als er den Charakter des Raumes mitbestimmen hilft. Es geht in der Geographie nicht um den Stoff an sich, nicht um seine physikalischen, chemischen oder psychologischen Eigenschaften, sondern um die Konsequenzen dieser Stoffeigenschaften für das Raumganze. So ist beispielsweise das Wetter nicht von seinen physikalischen Mechanismen her, sondern in seiner Rolle, die es unter anderen Elementen spielt, zu verstehen. Mit allen anderen Stoffbereichen verhält es sich ebenso, und der geographische Raum ist dann vollständig beschrieben, wenn alle maßgebenden stofflichen Komponenten berücksichtigt sind, was eben heißt, wenn die beinahe grenzenlose Stoffülle einbezogen ist.

Mit dem Hinweis auf die Art und Weise der Stoffbehandlung in der Geographie muß der Begriff der Stoffülle nun noch in einem neuen Licht gesehen werden. Wäre er der alles umfassende Begriff, wie er zunächst hätte verstanden werden können, würde er das Schulfach Geographie entweder zu einem im Ernst nicht zu bewältigenden Fach oder aber zu einem oberflächlichen Sammelfach von überall

Dr. Otto Wernli, Sengelbachweg 15, 5000 Aarau 
entlehnten Weisheiten stempeln. Er ist aber dadurch, daß alle stofflichen Gegebenheiten nur im Blick auf eine höhere Ganzheit ausgewertet werden, durchaus überschaubar und auch spezifisch geographisch gefärbt. In jeder Schulstunde liegt allerdings die Gefahr einer ungeographischen Optik sehr nahe, die Gefahr des Sich-Verlierens in reinen Sachdarstellungen. Sie besteht vor allem bei jenen Stoffgebieten, für deren Behandlung kein eigenes Schulfach existiert. Dann fühlt sich die Geographie verpflichtet, Auskunft zu geben. Wenn sie im Bewußtsein einspringt, daß vorübergehend der eigentliche Boden der Geographie verlassen wird, ist dagegen nichts einzuwenden; doch wenn die Grenzen unscharf werden, entsteht leicht ein gestaltloses Gerede. Die Gefahr des gleichzeitigen Umherschweifens in unterschiedlichsten Sach- und Denkkategorien verringert sich aber, wenn sich der Lehrer einem klaren geographischen Gesamtziel verpflichtet fühlt.

In den Lehrplänen sind geographische Ziele formuliert, in der Regel allerdings zu pauschal und darum zu wenig wirksam. Es genügt zum Beispiel nicht, festzustellen, im Geographieunterricht sei die Erdoberfläche zu beschreiben. Auch andere übliche Formulierungen, etwa von der Art: der Schüler muß die wichtigsten Länder kennen; das Mosaik der Landschaften und Völker ist zu beschreiben; der Geographieunterricht hat zur Erweiterung des räumlichen Horizontes beizutragen, reichen nicht aus. Und sogar gefährlich sind Zielsetzungen, die aus einer Aufzählung von Einzelaufgaben bestehen, weil damit geradezu zu isolierten Sachdarstellungen aufgefordert wird. Die Gezeiten, den Golfstrom, den Vulkanismus oder den Bergbau erklären, heißt doch, ein bißchen Astronomie, ein bißchen Geophysik und etwas Geologie nebeneinander behandeln, und nicht, das geographisch Ganze suchen. Bewährt hat sich folgende Zielumschreibung, deren allgemeiner Charakter in den weiteren Ausführungen allerdings noch den einzelnen Schulstufen entsprechend ergänzt werden muß: Es ist die Aufgabe der Geographie, ein objektives und einigermaßen ganzheitliches Weltbild aufzubauen, das einen fünffachen Zweck erfüllt.

1. Es muß in erster Linie ein Grundgerüst mit den nötigen Sachkategorien bereitstellen, damit wir die
Vielfalt der geistigen und materiellen Erscheinungen auf der Erde jederzeit logisch einordnen können. Es geht also vorab darum, einen Weg zu zeigen, der von den Einzelerscheinungen über ihre gegenseitigen Beziehungen zur sinnvollen Ganzheit führt.

2. Es muß auch das Grundgerüst für die räumlich richtigen Vorstellungen enthalten. Die relative Lage der Erscheinungen, Höhenunterschiede, Strecken und Flächen sind wichtige Komponenten, und es gilt, auch sie kategorial zu bestimmen. Diese Raumgrößen sollen in ihren möglichen Bedeutungen durch immer wiederkehrende Übungen als Kategorien erfaßt werden.

3. Kategorien haben vorwiegend formalen Charakter. Sie sind Gefäße, in die wir geistig die vielfältige Wirklichkeit abfüllen können, um sie dann vergleichend zu überblicken. Die Gefäße selber sind Bestandteil eines Weltbildes, darüber hinaus aber selbstverständlich auch ihre von Ort zu Ort variierenden Inhalte. Es genügt jedoch, eine typische Auswahl der Inhalte kennenzulernen, die für das Ganze des Weltbildes repräsentativ ist. Ganzheitlichkeit ist ja nicht gleichbedeutend mit Vollständigkeit; auch das stofflich noch unvollständige Ganze hat Ganzheitscharakter.

4. Aus dem Weltbild muß unsere eigene relative Situation und die Situation anderer feststellbar sein. Für den Schüler wie später für den Erwachsenen ist wichtig zu wissen, wo und wie er im Ganzen seines Weltbildes steht.

5. Jedes Weltbild beeinflußt das Denken und gibt Impulse für das Handeln. Der Aufbau eines geographischen Weltbildes soll das Denken über die Stoffund Fachgrenzen fördern und als eine objektive Grundlage für das Handeln in der geographischen Umwelt dienen.

\section{Das Verhältnis Länderkunde - Allgemeine Geographie}

Im Fachgebiet der Länderkunde werden begrenzte geographische Teilräume dargestellt. Die Allgemeine Geographie beschäftigt sich mit geographischen Einzelproblemen. Üblicherweise werden beide Arbeitswege benützt, und zwar so, daß die 
Länderkunde den Rahmen setzt und die Einzelprobleme innerhalb dieses Rahmens ihren Platz bekommen. Die Behandlung der Alpenländer eignet sich dazu, eingehender vom Problem des Fremdenverkehrs zu sprechen, und wenn der Orient an der Reihe ist, besteht Gelegenheit, die Ölprobleme in den Vordergrund zu stellen. Die Frage ist nun aber, ob dieses Vorgehen der eben formulierten geographischen Zielsetzung entspricht.

Vorausgesetzt, da $ß$ man mit dieser oder einer ähnlichen ganzheitlichen Zielsetzung der Geographie einverstanden ist, wird einem die übliche Methode eher-als Umweg denn als praktisches und direktes Mittel zur Lösung der Aufgabe erscheinen. Das länderkundliche Vorgehen begrenzt das Gesichtsfeld von Betrachtung zu Betrachtung anders, vergröbert oder verfeinert je nach der Einstufung des Landes den Maßstab und setzt entsprechend dem von einem Land $\mathrm{zu}$ erwartenden Gehalt immer wieder andere methodische Akzente. Man arbeitet also mit stets wechselnder Orientierung und von Fall zu Fall anders ausgerichtetem Denken, weshalb vielleicht letzten Endes ein künstlich zusammengesetztes, aber kein Weltbild aus einem Guß entsteht. Anders verhält es sich, wenn das Unterrichtsziel mit Überzeugung in der Länderdarstellung gesehen wird. Dann dürfen und müssen sogar divergierende Einzelbilder geschaffen werden. Die Betonung liegt dann aber auch nicht auf einem ganzheitlichen Denken, dessen Schulung als zentrale Aufgabe der Geographie hervorgehoben worden ist, sondern auf dem Erkennen von stofflichen Tatsachen und bestenfalls noch auf den Begründungen dafür, warum diese Tatsachen in einem Land gerade so und in einem andern Land anders geartet sind. Und zu fragen ist vielleicht einmal, ob Länderbilder den Schüler wirklich zu interessieren vermögen. Wirtschaftsfachleute, Politiker, Entwicklungshelfer benötigen eine Kenntnis jener Länder, mit denen sie beruflich zu tun haben, es gibt jedoch kaum eine Rechtfertigung dafür, daß der Schüler dieselben Interessen wie diese Fachleute und erst noch für eine größere Anzahl Länder aufbringen soll. Ein ganzheitliches Weltbild tangiert jedenfalls die menschliche Sphäre mehr als ein Mosaik von Länderbildern. Länderbilder sind sachbezogener als ein Weltbild; sie verpflichten zur Kenntnisnahme, zum Lernen, während ein Weltbild eher Denkanstöße gibt, zu Einsichten in die menschliche Situation im allgemeinen führt und mehr die Kategorien als die Fakten hervorhebt.

Die Allgemeine Geographie scheint die Fachrichtung zu sein, die sich für den Aufbau eines Weltbildes eignet, und die Erfahrung zeigt erst noch, daß sich der Schüler für sie wesentlich stärker erwärmen kann als für die Länderkunde. Darum muß eine Gewichtsverlagerung im Unterricht von der Länderkunde auf die Allgemeine Geographie befürwortet werden. Entscheidend ist allerdings, ob es gelingt, aus den allgemeinen Problemen jene Aspekte herauszuholen, die das gesuchte Weltbild mitbestimmen helfen. Tatsächlich wäre es viel naheliegender und auch bedeutend einfacher, die Probleme für sich zu klären, also beispielsweise Ebbe und Flut des Meeres als Ergebnis physikalischer Kräfte zu deuten, statt zu untersuchen, inwiefern diese Erscheinung für uns Erdbewohner wirklich von Bedeutung ist. Für die quasi probleminterne Sicht stünde auch mehr und besser geordnete Literatur zur Orientierung zur Verfügung; für die geographische Auswertung müssen Anhaltspunkte oft recht mühsam aus lokalen länderkundlichen Arbeiten zusammengetragen werden. Und eine besondere Schwierigkeit entsteht vor allem dadurch, daß man es ganz ohne die probleminterne Sicht häufig auch nicht machen kann. Wenn schon von Ebbe und Flut gesprochen wird, sollte der Schüler doch auch verstehen, warum es diese Erscheinung gibt. Nun aber richtig abzuwägen, wie weit ausgeholt werden soll und ausgeholt werden darf, ohne die eigentliche geogragraphische Darstellung in ihrem Zusammenhang $\mathrm{zu}$ beeinträchtigen, ist außerordentlich schwierig. Vor allem wichtig ist jedenfalls, daß das zu schaffende Weltbild nie durch Anhängsel aller Art so belastet wird, daß statt dem erwünschten ganzheitlichen Gesicht eine innerlich unzusammenhängende Anreihung von Wissen verschiedenster Herkunft entsteht. - In bezug auf die Länderbilder ist vielleicht noch zu sagen, daß sie bei Bedarf auch auf dem Weg über die Allgemeine Geographie geschaffen werden können. Sie entstehen wohl nicht mehr in einem Zug, sondern ergeben sich allmählich mit der Besprechung all jener Probleme, die für ein und dasselbe Land typisch sind, möglicherweise sind sie aber sogar markanter als jene aus der länderkundlichen Geographie. 


\section{Das Prinzip «vom Nahen zum Fernen»}

Für die Planung eines sinnvollen Geographieunterrichts gibt es leider nur wenige methodische Richtlinien. Der Lehrer ist vorwiegend auf seine eigenen Ideen angewiesen. Wenn er ein besonderes Interesse am Fach hat und vor allem auch die nötige Zeit für die Planung aufbringt, werden überzeugende Lektionen entstehen. Doch auch dann noch sind es individuelle und von Schule zu Schule anders geartete Leistungen, weshalb es beispielsweise schwerhält, Vergleiche zu ziehen und eine allgemein gültige Gestalt des Faches zu erkennen. Nicht zufällig sind darum noch die abstrusesten Vorstellungen vom Wesen der Geographie im Schwange. Vermutlich das geläufigste Ordnungsprinzip der Geographie ist das Prinzip "vom Nahen zum Fernen». Fast alle Lehrpläne nehmen darauf Rücksicht, indem sie vorschreiben, zunächst von der engeren Heimat des Schülers, dann vom Wohnkanton, vom Land, von den umliegenden Ländern, von Europa und schließlich von den näheren und weiteren anderen Kontinenten zu sprechen. Genau besehen dient aber gerade dieses am häufigsten verwendete Prinzip nicht der erwünschten Unterrichtsstruktur. Es ist ein summatives Prinzip ohne innere Logik, und vielleicht sogar ein verkehrtes Prinzip. Statt zu zeigen, wie die einzelnen Bausteine von Stunde zu Stunde aufeinander gelegt werden können, um so eine von unten nach oben abgestimmte Ganzheit zu erreichen, erlaubt dieses Prinzip ein fast zusammenhangloses Nebeneinander von vielerlei Einzelbauten. Leichtere und schwierigere Stoffgebiete erscheinen zudem in bunter Mischung; eine Anpassung des Schwierigkeitsgrades an den Entwicklungsstand des Schülers ist nicht im Plan. Und geradezu als verkehrt erscheint das Prinzip deshalb, weil der Schüler sehr früh schon ein Interesse an der fremden Welt hat und er seine nähere Umwelt mit einem differenzierteren Maßstab betrachten könnte, wenn er vorher hätte herumschauen dürfen. Selbstverständlich ist es richtig, wenn die ersten geographischen Begriffe durch direkte Anschauung in der näheren Umgebung gewonnen werden, doch wäre es sinnvoll, im weiteren Unterricht die meistens grobzügiger behandelten Probleme der Ferne vorweg zu nehmen. Nachher sollte allmählich vom Fernen zum Nahen zurückgekehrt werden, mit stetig wachsenden Ansprüchen an die Betrachtungsweise. Gelegentlich könnte auch ein kurzfristiges Hin und Her zwischen Objekten in der Ferne und Objekten in der Nähe aufschlußreich sein.

Während das Prinzip «vom Nahen zum Fernen» die Reihenfolge der geographischen Themen für den gesamten Unterricht festlegt, zeigt ein zweites Ordnungsprinzip, das "länderkundliche Schema», wie sich die Arbeit innerhalb eines begrenzten Themas gliedern läßt. Das länderkundliche Schema hat immer einen Raum zum Gegenstand, und dieser soll hintereinander nach seiner Lage, nach der Größe, dem Relief, den Bodenverhältnissen, dem Klima, der Bewässerung, nach seinen Erzeugnissen und nach den Bewohnern untersucht und dargestellt werden. Eine gewisse Freiheit zu variieren besteht, doch ist darauf zu achten, da $\beta$ in jedem Fall die vorhandenen kausalen Zusammenhänge aufleuchten. Die Erfahrung zeigt nun allerdings, daß trotz der zu berücksichtigenden Kausalität zwischen den einzelnen Raumaspekten selten ganzheitliche Raumbilder entstehen. Die Räume erscheinen, man kann es drehen wie man will, als mehr oder weniger ungestaltete Ausschnitte aus der geographischen Stoffülle. Und im Rahmen der vorliegenden Betrachtung ist dieses Ordnungsprinzip ohnehin nicht von Bedeutung, weil es höchstens der Länderkunde, nicht aber der Allgemeinen Geographie auf dem Weg zu einem ganzheitlichen Weltbild dient.

Es bleibt noch der Hinweis auf die heute häufig zu hörende Formel vom «Mut zur Lücke». Tatsächlich benötigt die Geographie diesen Mut in besonderem Maße, weil es niemals nur annähernd möglich wäre, Vollständigkeit zu erreichen. Gerade darum aber, weil es ohne Lücken sowieso nicht geht, handelt es sich um eine nichtssagende Formel. Hilfreicher wäre eine verantwortungsbewußte Aussage darüber, wo ohne Bedenken Lücken offen gelassen werden dürfen, oder mit anderen Worten, mit welcher Stoffauswahl ein ganzheitliches Weltbild zu erreichen ist.

\section{Wege zum Ziel}

Es scheint mindestens fünf Möglichkeiten zu geben, die geistigen und materiellen Erscheinungen auf der Erde zu einem ganzheitlichen geographischen 
Weltbild zu vereinen. Diese fünf Möglichkeiten sollen in der Folge kurz dargestellt werden. Selbstverständlich führen daneben auch philosophische und religiöse Einsichten zu Weltbildern, doch unterscheiden sie sich vom geographischen Weltbild dadurch, daß sie hinter den Tatsachen den Sinn zu bestimmen versuchen. Das geographische ist ein vordergründigeres, stoffliches, nicht interpretiertes Weltbild. Auch naturwissenschaftliche Weltbilder sind nochmals etwas anderes. Sie stellen Querschnitte durch die Tatsachenwelt dar, wie sie bei Anwendung bestimmter Untersuchungsmethoden sichtbar werden.

1. Die einfachste, aber auch die am wenigsten integrierte geographische Zusammenschau ist die Summation. Sie ist vergleichbar mit dem Inventar eines Geschäftsbetriebes. Durch geeignete Stoffgruppierungen erreicht man überschaubare Verhältnisse, gewinnt man Einblick in die Kategorien, Mengen, gegenseitigen Relationen und Grenzen der vorhandenen Gegebenheiten. Die summative Betrachtung ist jedoch, wie schon gesagt und wie es auch die dafür gewählte Bezeichnung zum Ausdruck bringt, von schwacher Bindungskraft. Es ist auch keinesfalls möglich, den gesamten Geographieunterricht $\mathrm{zu}$ einer nur summativen Weltbetrachtung werden zu lassen. Kapitelweise, entweder als Bestandesaufnahme zur Einführung oder vielleicht eher noch als Zusammenfassung hintenher kann der summative Aspekt einen gewissen Wert haben.

2. In viel höherem und unter sich in gleichem Maße wertvoll sind die vier weiteren ganzheitlichen $\mathrm{Be}$ trachtungsweisen. Eine davon ist die genetische. Die Erfahrung ergibt, daß es für Schüler und Lehrer sehr befriedigend ist, die für unser Weltbild maßgebenden Elemente aus der Erdgeschichte herzuleiten. Man darf indessen der Versuchung nicht nachgeben, die erdgeschichtlichen Epochen als solche auszumalen. Die Linie nach vorne zum heutigen Weltbild muß strikte eingehalten werden, und wenn das gelingt, entsteht ein Ganzheitseindruck von hoher Komplexität. Die Methode eignet sich weniger für die Volksschul- als für die Mittelschulstufe, jedoch auch in der Mittelschule, wie noch zu zeigen ist, am besten in Kombination mit anderen Methoden.

3. Ein weiterer Weg zum gesetzten Ziel ist das Aufsuchen der kausalen und finalen Beziehungen zwi- schen den einzelnen Stoffgruppen. Das Gesamtergebnis ist ein Wirkungsgefüge aus vielseitigen $\mathrm{Ab}$ hängigkeiten. Es ist einem geknüpften Teppich vergleichbar, der daraufhin untersucht worden ist, aus wievielen Fäden, aus welchen Fadenarten und wie die einzelnen Knöpfe geknüpft worden sind. Die kausal-finale Methode entspricht unserem üblichen Denken am besten. Sie setzt jedoch eine große Menge von speziellen Kenntnissen aus anderen Fächern voraus, wenn sie nicht nur oberflächlich gehandhabt werden soll. Für den Schüler der Volksschulstufe ist sie schwer und auch für den Mittelschüler noch recht anspruchsvoll. Er sollte sich vorweg schon in den physikalischen, chemischen, astronomischen und völkerpsychologischen Grundlagen auskennen.

4. Eine vierte Methode kann als funktionale Methode bezeichnet werden. Durch die organisatorische Tätigkeit des Menschen ist auf der Erde eine Art künstliches Wirkungsgefüge geschaffen worden. Es gibt weltweite wirtschaftliche, politische, wissenschaftliche, technische und geistige Abhängigkeiten, für die der Mensch selber verantwortlich ist. Diesen Abhängigkeiten gilt es auch nachzuspüren, und das dabei gewonnene Bild ist ein neuer ganzheitlicher Aspekt der stofflichen Substanz der Geographie. Es ist vermutlich ohne weiteres ersichtlich, $\mathrm{da} ß$ diese stoffliche Substanz für alle Betrachtungsweisen dieselbe bleibt, daß jedoch von Betrachtungsweise zu Betrachtungsweise andere Kombinationen aufleuchten. Im Unterricht hat man es also nicht, ob man nun die Methode zwei, die Methode fünf oder die Methode drei anwende, mit immer wieder anderen stofflichen Gegebenheiten zu tun, jedoch mit anderen Querschnitten durch den Stoff, das heißt mit anderen Betonungen im Weltbild. Es wird also stets stark von der Absicht abhängig sein, welche Methode wir anwenden. Soll ein möglichst vollständiges und nicht ein einseitiges Weltbild entstehen, sind alle ganzheitlichen Methoden in sinnvoller Abgewogenheit einzusetzen. Leider reicht dazu die zur Verfügung stehende Zeit in der Regel nicht aus.

5. Für alle Schulstufen in gleicher Weise geeignet ist die normative Methode. Es ist die Methode, die von bestimmten Weltbild-Vorstellungen ausgeht und die geographische Wirklichkeit damit konfrontiert. 
Aus dem Vergleich ergeben sich positive und negative Abweichungen, die das realistische Weltbild kennzeichnen. Vorweg müssen in der Vorstellung die Normen als die Bezugsmaßstäbe geschaffen werden. Schon allein diese Unterrichtstätigkeit ist überaus anregend, und sie läßt im Schüler jene Erwartungen aufkommen, die ihn nachher zum selbstverständlichen Dabeisein veranlassen. Im übrigen stellt die normative Betrachtung in der erwünschten Weise den Menschen ins Zentrum; nicht im falsch verstandenen Sinn den Menschen, über den man wie über ein anderes Objekt auch noch redet, sondern den Menschen mit seinen Vorstellungen und Bedürfnissen. Zunächst sind es die Vorstellungen und Bedürfnisse des Schülers, dann aber bald auch die Vorstellungen und Bedürfnisse des Menschen mit anderer Denkweise, von anderer Rasse und mit anderer Religion. So erhält die Geographie den sie vertiefenden und immer wieder geforderten anthropologischen Bezug. Für die geographische Betrachtung eignen sich Normen verschiedenster Art. Am nuanciertesten wird das Weltbild dann, wenn mehrere möglichst unterschiedliche Normen verwendet werden. Es sollte geradezu zu einem Spiel mit Normen kommen.

\section{Der Unterricht auf der Volksschulstufe}

Als Gesamtziel des Geographieunterrichts kann für alle Schulstufen das ganzheitliche Weltbild gelten, doch muß es, dem sich erweiternden Bewußtsein des heranwachsenden Menschen entsprechend, von Stufe zu Stufe vertieft und vervollständigt werden. In den ersten zwei, drei Jahren des dannzumal noch betont heimatkundlichen Geographieunterrichts, also in der dritten, vierten, fünften Primarschulklasse, ist bestimmt das Herleiten von einigermaßen klaren Grundbegriffen und einfacheren landschaftlichen Zusammenhängen das Richtige. Darüber soll hier nicht ausführlicher gesprochen werden, weil es sich bei diesem Unterricht noch nicht um Geographie im eigentlichen Sinne handelt. Das heißt allerdings nicht, daß das, was auf dieser Stufe als Grund gelegt wird, nicht von entscheidender Bedeutung für allen späteren Unterricht sein kann. Es verhält sich damit wie mit dem Einmaleins, das bis weit hinauf in der Schule Sand ins Getriebe der Mathematik bringen kann, wenn es nie seriös gelernt worden ist. Auf der Oberstufe der Volksschule setzt der Geographieunterricht im umfaßenden Sinn ein, und für diese Stufe als zentrale Methode geeignet ist die bereits kurz dargestellte normative Betrachtungsweise. Für die Planung des gesamten geographischen Arbeitsprogramms der Oberstufe ist darum zunächst eine Übersicht über die Bedürfnisse und Wünsche des Menschen der geographischen Umwelt gegenüber erforderlich. Es zeigt sich, daß Hauptbedürfnisse und Teilbedürfnisse unterschieden werden können und letzten Endes eine ganze Bedürfnishierarchie als vielgliedriger Maßstab herleitbar ist.

Alle umweltbezogenen Anliegen des Menschen lassen sich in drei Gruppen einteilen: in die Gruppe der primären, existenzialen Anliegen, in die Gruppe der wirtschaftlichen Anliegen im weitesten Sinn, das heißt all jener Bedürfnisse, die für die Entfaltung, für die Aktivität des Menschen verantwortlich sind, und in die Gruppe der kulturellen, der seelisch-geistigen Anliegen. In jeder Gruppe kann eine Hauptnorm für die geographische Betrachtung gesehen werden. So entsteht von den Betrachtungsweisen her eine Dreiteilung der normativen Geographie. Stofflich sind die Teile genügend umfangreich, um zu Jahrespensen zu werden, die als Existenzgeographie, Wirtschaftsgeographie und Kulturgeographie zu bezeichnen wären. In der Existenzgeographie wird die geographische Welt im Lichte der primären Lebensbedürfnisse betrachtet; die Wirtschafts- und die Kulturgeographie gehen in entsprechender Art von den wirtschaftlichen und kulturellen Bedürfnissen des Menschen aus.

Ohne Symmetriezwang lassen sich im Bereich der drei Hauptnormen je vier Teilnormen finden, die die Jahrespensen in Quartalspensen gliedern, und schließlich können auch diese Teilnormen bis hinunter zu den einzelnen Lektionen noch mehrfach zerlegt werden. Hier seien von allen Stufen bloß noch die Quartalspensen genannt, voraus das maßgebende Bedürfnis, mit dessen Hilfe die Norm zu definieren ist, dahinter die Titel für die jeweilige geographische Entsprechung: 


\section{Existenzgeographie}

Bedürfnis nach Abwechslung - Tageslauf und Jahreslauf Bedürfnis nach Gesundheit - Gesundheit und Krankheit Bedürfnis nach Geborgenheit - Wohnstätten, Dörfer, Städte Nahrungsbedürfnis

- Wie man sich ernährt

Wirtschaftsgeographie

Bedürfnis nach den Lebens- - Erwerb der lebensnotnotwendigen Mitteln Bedürfnis nach Rohstoffen Bedürfnis nach Energie Bedürfnis nach einer guten Umweltorganisation

\begin{tabular}{|c|c|}
\hline ulturgeographie & \\
\hline Ästhetisches Bedürfnis & $\begin{array}{l}\text { - Das Schöne in natürlichen } \\
\text { Umwelten }\end{array}$ \\
\hline Gestaltungsbedürfnis & $\begin{array}{l}\text { - Praktische Umwelt- } \\
\text { gestaltung }\end{array}$ \\
\hline $\begin{array}{l}\text { Bedürfnis nach geistiger } \\
\text { Entfaltung }\end{array}$ & - Bildung und Unterhaltung \\
\hline $\begin{array}{l}\text { Bedürfnis nach einer ange- } \\
\text { nehmen geistigen Atmosphäre }\end{array}$ & - Kulturtypen \\
\hline
\end{tabular}

Damit sind drei Jahrespensen grob charakterisiert. Es fehlt noch jenes Pensum, das von der heimatkundlichen Unter- und Mittelstufengeographie zur normativen Geographie der Oberstufe überleitet. Das kann ein Jahrespensum oder auch ein gekürztes Pensum mit geographischen Problemen aus dem Gebiet der Schweiz sein. Eine sinnvolle Überleitung ist gewährleistet, wenn die ausgewählten schweizerischen Gegebenheiten zuerst noch heimatkundlich beschrieben, in einem zweiten Durchgang dann aber bereits einer einfachen normativen Betrachtung unterstellt werden. Die Arbeit entspricht der bisherigen Schweizergeographie des ersten Oberstufenjahres, mit einem engeren Stoffbereich allerdings, andererseits mit einer vertieften Durchdringung des ausgewählten Stoffes. Auszuwählen sind die Themen so, daß eine gewisse Vollständigkeit durch die Ausgewogenheit repräsentiert wird. Eine umfassende Schweizergeographie ist deshalb nicht anzustreben, weil in die Allgemeine Geographie der folgenden Jahre auch immer wieder schweizerische Beispiele einbezogen werden.

Mag sein, daß man die Beschränkung des Faches in den Schuljahren der Oberstufe auf die normative
Sicht als Einengung empfindet, und darum sei hier nochmals auf die Tatsache hingewiesen, daß eben jede der fünf genannten ganzheitlichen Betrachtungsweisen von der summativen bis zur normativen über den gesamten geographischen Stoff verfügt. Alle Betrachtungsweisen sind ganzheitliche Betrachtungsweisen. Sie unterscheiden sich im Weg; im Ziel nur insofern, als sie es von verschiedenen Seiten erreichen und es darum in immer wieder anderem Licht erscheint. Im Stoff aber unterscheiden sie sich gar nicht. Die allenfalls befürchtete Einengung ist auch deshalb nicht vorhanden, weil die nicht normativen Betrachtungsweisen, vor allem die kausale und die genetische, stets auch zum Zuge kommen. Die "Warum-Frage» läßt sich selbstverständlich auch in der normativen Geographie nicht umgehen, und zu ihrer vollständigen Beantwortung müssen kausal-finale, genetische, funktionale und summativ-statistische Erklärungen gesucht werden. Mit der Wahl der normativen Betrachtungsweise ist aber darüber entschieden, in welcher Richtung die mannigfaltige stoffliche Substanz der Geographie durchlaufen wird, mit anderen Worten: nach welchen Prinzipien der Unterricht aufgebaut werden soll. Die kausale Betrachtungsweise wie auch die anderen Betrachtungsweisen werden nicht als $\mathrm{Me}$ thoden zur Sicherung einer klaren Linie im Unterricht beigezogen, sondern sie dienen der Beantwortung einzelner Fragen, die sich aus der normativen Sicht ergeben.

\section{Der Geographieunterricht in der Mittelschule}

Eine Erweiterung und Vertiefung des Geographieunterrichts ist nun noch in der Art möglich, daß alle Betrachtungsweisen mit einigermaßen gleichem Gewicht hintereinander zur Anwendung kommen. Das heißt, daß außer dem normativen Durchgang durch den Stoff auch ein genetischer, ein kausalfinaler und ein funktionaler Durchgang vollzogen werden. Der normative selber wird gegenüber der Volksschul-Oberstufe verfeinert. Wie schon gesagt, hat eine systematisch summative Betrachtung nicht viel Sinn, doch leisten summative Stoffgruppierungen als Kapitel-Zusammenfassungen oder bei der Einführung in ein neues Kapitel gute Dienste. 
Die Erfahrung zeigt, daß die Verwendung aller Betrachtungsweisen einen anregenden und überaus gehaltvollen Geographieunterricht ergibt. Auf natürlichem Weg gliedert sich das ganze Stoffgebiet in gut überschaubare und auch leicht vergleichbare Abschnitte. Lehrer und Schüler wissen in jedem Zeitpunkt, an welcher Stelle im ganzen Plan eine Arbeit ihren Platz hat, was erledigt ist und was noch bevorsteht. Es ergeben sich Wiederholungen, auch Wiederholungen durch das Zurückkommen auf schon besprochene räumliche Gebiete, doch immer unter anderen Gesichtspunkten, so daß einem die Tatsachen häufig schon zum vornherein als vertraut erscheinen, man dann aber doch ständig zusätzliche Nuancen erkennt. Aller Stoff fängt an durchsichtig zu werden; er läßt sich drehen und von mehr als einer Seite anschauen, und er bekommt, wie es sein sollte, seinen Sinn als Mittel zum Zweck. Erreicht werden soll ja ein ganzheitliches Weltbild, das dem heranwachsenden jungen Menschen bei der Lebensbewältigung Orientierungshilfe sein kann. Der Stoff weist bei diesem Vorgehen tatsächlich über sich selbst hinaus. Dadurch, daß das Weltbild mehr als einmal geschaffen wird und nicht als ein schwer zu erreichendes Ziel am Ende der ganzen Schulzeit steht, ist die Erwartung beim Schüler größer. Aus diesem Grunde hat er auch ein stärkeres Interesse am Fach. Da das Weltbild zudem von Durchgang $z u$ Durchgang in anderem Licht erscheint, ist man zu Vergleichen veranlaßt, was zu einer Rückschau zwingt und zur erwünschten Vertiefung des Erreichten beiträgt. Schließlich darf erwähnt werden, daß die Betrachtungsweisen in der aufgeführten Reihenfolge, die genetische am Anfang und die verfeinerte normative am Schluß, eine allmähliche Steigerung der Ansprüche an den Schüler mit sich bringen, womit ein weiteres ordnendes Element in den Geographieunterricht kommt.

Die genetische Betrachtung, also die Herleitung des heutigen Weltbildes aus der Erdgeschichte, wird fraglos als die passende Einführung in die Mittelschulgeographie empfunden. In diesem Kapitel liegen die Betonungen auf den astronomisch-kosmogonischen, den geologischen und den morphologischen Gegebenheiten. In engem Zusammenhang mit den geologischen und morphologischen Tatsachen läßt sich auch von den durch sie bedingten wirtschaftlichen Grundlagen sprechen. Doch auch die Entwicklung des Lebens bis und mit dem Auftreten des Menschen, der Aufgliederung der Menschheit in die alten und die heutigen Menschenrassen und der Ausbreitung des Menschen über die Erde im Laufe des ganzen Quartärs muß dargestellt werden. Als summative Zusammenfassungen kommen geschichtliche Kontinents- und Länderbilder in Frage.

Für die nun folgende kausal-finale Betrachtung sei vorgeschlagen, sie mit der funktionalen Betrachtung zu vereinen. Beide Methoden berühren sich an so vielen Stellen, daß es angezeigt erscheint, sie miteinander zu verwenden. Gesucht wird mit beiden das Wirkungsgefüge, das durch mehrfache Abhängigkeiten der stofflichen Elemente unter sich entsteht, und der Unterschied liegt nur noch darin, daß die eine Methode die kausalen und finalen und die andere die durch menschliche Organisation geschaffenen Abhängigkeiten hervorhebt. In diesem zweiten Kapitel der Mittelschulgeographie erscheinen meteorologische und klimatologische Themen im Vordergrund. In erster Linie verantwortlich für das Wirkungsgefüge ist ja die Sonnenenergie, und sie in ihrer lokalen Verteilung bestimmt den Wetterund den Klimacharakter, wovon tausenderlei irdische Erscheinungen abhängig sind. Das durch den Menschen geschaffene Wirkungsgefüge ist selbstverständlich auch sehr stark durch das Klima bedingt, doch spielen menschliche Überlegungen aller Art eine wichtige zusätzliche Rolle. Auch in diesem Kapitel sind summative Kontinents- und Länderbilder sinnvolle Teilziele.

Mit der normativen Sicht endlich kann die stoffliche Substanz der Geographie ein drittes Mal im Blick auf ein Weltbild aufbereitet werden. Es ist nun aber nicht mehr nötig, wie in der Volksschule, mehrere Teilnormen einzeln zu verwenden. Beim Schüler kann jetzt bereits mit einem gewissen Überblick gerechnet werden, und dem Alter entsprechend besitzt er die Fähigkeit, mit einem allgemeineren menschlichen Maßstab zu messen. Es ist nun möglich, die nähere und weitere geographische Umwelt im Lichte des Existenzbedürfnisses der ganzen Menschheit zu betrachten, eines Bedürfnisses materieller und geistiger Art. Dabei entsteht das konzentrierteste und darum auch aussagekräftigste Weltbild. Es erlangt für den Schüler die Bedeutung einer Zusammenfassung aller vorangegangenen Bemü- 
hungen auf höchster Ebene, und es bildet darum auch einen befriedigenden Abschluß des mehrjährigen Geographieunterrichts. Es ist insofern auch ein verfeinertes Weltbild gegenüber jedem vorher erreichten, als es die wesentlichen Züge am stärksten hervorhebt und die untergeordneten Sachverhalte an den ihnen gebührenden Platz verweist.

\section{Pädagogische Forderungen}

Wenn man sich auf das Wesen eines Schulfaches besinnt, vergißt man leicht den Schüler, für den allein das Fach da ist. Man untersucht die stoffliche Seite des Faches und denkt nicht daran, da $\beta$ der Stoff erst in Verbindung mit den Bedürfnissen des Schülers das Fach ausmacht. Für die Wissenschaft ist ein Stoff an sich wichtig, doch das Schulfach ist ein verwobenes Gebilde aus stofflichen und menschlichen Belangen. Der Schüler soll am Stoff wachsen; das heißt, daß der Schüler über den Fachcharakter bestimmt. Aber richtig ist auch die andere Forderung, da $B$ der gegebene Stoff vom Schüler verstanden werden muß, und das heißt, daß auch der Stoff ernst zu nehmen ist. Diese Feststellungen gelten für alle Schulstufen, auch für die Mittelschule.

In dieser Stellungnahme zur Geographie ist der Schüler bis dahin zwar nicht ganz vergessen geblieben, doch lassen sich beim Gedanken an ein wirklich ausgeglichenesFach gleichwohl noch weitere Ansprüche von der Schülerseite her rechtfertigen. Werden sie berücksichtigt, vertiefen sie erfahrungsgemäß den Unterricht. Vielleicht die wichtigste weitere Forderung ist jene nach einer geringeren Belastung des Gedächtnisses. Es liegt gar nicht im Wesen der Geographie, daß sie das Schülergedächtnis mehr als andere Fächer belasten muß. Es ist wohl nur der einfachste und schnellste Weg, um in kurzer Zeit möglichst viele Tatsachen zu bewältigen, wenn man sie gedächtnismäßig lernen läßt. Auf die Dauer ist aber bestimmt nicht viel damit gewonnen, und es bedeutete eine gewichtige Fachaufwertung, wenn dafür die anderen menschlichen Kräfte, das Denken, die Phantasie, die Erlebniskraft und die zeichnerischhandwerklichen Fähigkeiten verstärkt und systematisch geschult werden könnten. Die Vielfalt des geographischen Stoffes eignet sich für die Ausbildung aller genannten Kräfte. Beim Denken ist es die synthetisierende Art, die der Geographie am besten entspricht. In den anderen Fächern steht normalerweise die analysierende Art im Vordergrund, und darum kann die geographische Denkschulung als eine notwendige Ergänzung zur übrigen Denkschulung angesehen werden.

Richtig ist bestimmt auch, wenn darauf Rücksicht genommen wird, daß Schüler verschiedenen Alters auf einen Themenkreis unterschiedlich ansprechen. Auffallend ist zum Beispiel, wie wetter- und klimakundliche Fragen in unteren Klassen auf wesentlich mehr Interesse stoßen als völkerkundliche Fragen, während weiter oben völkerkundliche Probleme normalerweise als spannende Themen empfunden werden und zu einem entsprechenden Schülereinsatz im Unterricht führen. Erfahrungen dieser Art können so ausgewertet werden, daß man sich beim Setzen von Unterrichtsschwerpunkten ebenso vom Entwicklungsstand des Schülers wie von stoffbezogenen Überlegungen leiten läßt. Man geht zu schematisch vor, wenn man allein eine klare Linie in den stofflichen Gegebenheiten im Auge hat.

Dann ist es in der Geographie wie in anderen Fächern auch so, daß eine gewonnene Erkenntnis erst voll zur Geltung kommt, wenn sie mehrfach erprobt und in unterschiedlichen Situationen angewendet worden ist. Es genügt nicht, Feststellung an Feststellung zu reihen in der Meinung, was einmal hergeleitet und formuliert sei, könne als bekannt und als erledigt angesehen werden. Wenn, wie für die Geographie gefordert, die Erkenntnisse Bestandteil eines Ganzen werden sollen, müssen sie ohnehin vom wachsenden Ganzen her immer wieder neu beleuchtet und relativiert werden. Wenn diese Forderung nach wiederholtem Betrachten ein und desselben Sachverhaltes nun auch noch pädagogisch motiviert werden kann, ist der Unterricht offensichtlich dann am ergiebigsten, wenn es gelingt, alle einmal erarbeiteten maßgebenden Einsichten ständig präsent und für neue Einsätze bereit zu halten. Nur unter dieser Bedingung scheint das geographische Weltbild entstehen zu können, und nur unter dieser Bedingung lernt der Schüler die geographischen Gegebenheiten unmißverständlich und auch mit der nötigen Tiefe kennen.

Schließlich sei noch auf die zwar längst nicht mehr unbekannte Tatsache hingewiesen, daß der Schüler 
in der Regel kein großes Interesse für reine topographische Feststellungen aufbringt. Es gibt auf allen Schulstufen Ausnahmen, Schüler, denen es leicht fällt, sich topographische Kenntnisse in großer Zahl anzueignen. Die Mehrzahl der Schüler jedoch nimmt das topographische Erdbild mühsam und am besten in Verbindung mit Erlebnissen auf. Selbstverständlich gehört die Vermittlung eines Gerüstes aus topographischen Kenntnissen zur Aufgabe der Schule, doch ist es angezeigt, die Schwierigkeiten zu berücksichtigen und nicht über die notwendigen Fixpunkte hinauszugehen und auch diese nur im Rahmen sinnvoller Zusammenhänge einzuführen. Es ist gut möglich, ein topographisches Erdbild so in das umfassendere geographische Weltbild zu integrieren, daß es als wirklich notwendiger Bestandteil erscheint und auch aufgenommen wird. Das topographische Erdbild kann wenigstens zum Teil auch über topographische Länderbilder aufgebaut werden, so daß auch das Ländermosaik der Erde, das durch das Umgehen der Länderkunde vernachläßigt wird, in angemessener Weise in Erscheinung tritt.

\section{Die Situation der Zeit und die Geographie}

«Die Wohlstandsprobleme sind einigermaßen gelöst - was nun?» Zu diesem Thema nimmt Prof. Dr. E. Küng von der Hochschule St. Gallen Stellung (Neue Zürcher Zeitung Nr. 524 vom 11. 11.73), und er legt der suchenden Jugend die Antwort in den Mund: "Was uns fehlt, ist ein abgerundetes Weltbild». Das Weltbild in dem von Prof. Küng verstandenen Sinn beinhaltet mehr als ein geographisches Weltbild, doch darf wohl gesagt werden, daß auch das geographische Weltbild Bestandteil des gemeinten Weltbildes ist. Die Jugend verlangt ein abgerundetes Weltbild, weil sie richtigerweise hofft, darin die Dimensionen für die Lebensorientierung zu finden. Viele der von ihr gesuchten Leitlinien entsprechen jenen unumstößlichen Sachverhalten, geistigen und materiellen, welche die Geographie mit ihren Betrachtungsweisen über die ganze Erde hinweg aufzeigt. Und zwar erscheinen diese Gegebenheiten, wie dargestellt worden ist, nicht in einem unverbundenen Nebeneinander, sondern als Bestandteil des Weltbildes aufeinander bezogen. Zu den geographischen Dimensionen kommen im umfassenden Weltbild viele andere hinzu, doch wird im Bereich der Geographie der notwendige stoffliche Boden gelegt. 